Appl. Phys. 5, $71-77$ (1975)

(C) by Springer-Verlag 1975

\title{
Electron Spin Polarization in Field Emission: Calculation of the Effects Due to External Fields
}

\author{
W. Eckstein and N. Müller \\ Max-Planck-Institut für Plasmaphysik, EURATOM-Association, D-8046 Garching, \\ Fed. Rep. Germany
}

Received 8 August 1974/Accepted 11 September 1974

\begin{abstract}
In field emission experiments with spin polarized electrons a magnetic field is superposed on the electric emission field to define the preferred spin direction. The motion of the polarization vector in these fields was calculated for rays emanating from individual points of the emitter by integrating the equation of motion and taking into account relativistic terms. There is a slight shift of the polarization vector from its initial direction. If the initial polarization is aligned with the magnetic field and the emission tip is sufficiently well centred in the magnetic field, the tilting of the polarization vector for a beam of electrons starting not too far from the tip apex is less than $10^{\circ}$.
\end{abstract}

Index Headings: Field emission of electrons - Electron spin polarization

In recent experiments electron spin polarization (ESP) of field emitted electrons has been detected [1-4]. The emitter in these investigations is located in a magnetic field which defines the preferred spin direction. In front of the emitter the electric field required for field emission (FE) is superposed on the magnetic field. If the ESP varies in a not undefined manner after the emission process, it is possible to investigate the spin distribution in the electronic states of the emitter or the spin dependence of the emission process. In this paper we report on some calculations of the ESP variation in the fields in front of the emitter for rays emanating from individual points of the emitter. Effects in the tunnel barrier are not taken into account. The calculations were made on the IBM $360 / 91$ at IPP.

\section{Description of the ESP Variation}

The ESP can be described by the polarization vector $\boldsymbol{P}$. For a single electron represented by a wave packet $|\psi\rangle \boldsymbol{P}$ is defined in the rest system by the expectation value of the polarization operator $\boldsymbol{\sigma}$ formed from the
Pauli matrices

$\boldsymbol{P}:=\langle\boldsymbol{\sigma}\rangle=\frac{\langle\psi|\boldsymbol{\sigma}| \psi\rangle}{\langle\psi \mid \psi\rangle}, \quad \boldsymbol{\sigma}=\left(\sigma_{x}, \sigma_{y}, \sigma_{z}\right)$.

Correspondingly, the average polarization vector of an ensemble of electrons in states $\left|\psi_{i}\right\rangle$ with nearly the same momentum (e.g. a beam of electrons) is given in the centre of mass system by

$$
\begin{gathered}
\boldsymbol{P}=\langle\boldsymbol{\sigma}\rangle=\frac{\sum_{i}\left\langle\psi_{i}|\boldsymbol{\sigma}| \psi_{i}\right\rangle}{\sum_{i}\left\langle\psi_{i} \mid \psi_{i}\right\rangle}=\frac{\sum_{i} N_{i} P_{i}}{\sum_{i} N_{i}} \\
\boldsymbol{P}_{i}:=\frac{\left\langle\psi_{i}|\boldsymbol{\sigma}| \psi_{i}\right\rangle}{\left\langle\psi_{i} \mid \psi_{i}\right\rangle}, \quad N_{i}:=\left\langle\psi_{i} \mid \psi_{i}\right\rangle,
\end{gathered}
$$

where $P:=|\boldsymbol{P}| \leqq 1$ is the degree of polarization, and $\hat{p}:=\boldsymbol{P} / \boldsymbol{P}$ is the preferred spin direction.

In electric and magnetic fields the vector $\boldsymbol{P}$ will move. If these fields are macroscopic, i.e. if their variation in space and time is small relative to the extent of a wave packet, a classical equation of motion is valid 
[5], i.e.

$\frac{d}{d t} \boldsymbol{P}(t)=-\boldsymbol{\omega} \times \boldsymbol{P}(t)$,

where $\omega$ is the spin precession vector, and $\omega=|\omega|$ is the spin precession frequency. If the motion of $\boldsymbol{P}$ is observed in the instantaneous rest system, which does not rotate relative to the laboratory system, and if the time $t$, the velocity $\boldsymbol{v}$, the electric field $\boldsymbol{E}$ and the magnetic induction $\boldsymbol{B}$ are measured in the laboratory system, according to [6], $\omega$ obeys the equations

$$
\begin{aligned}
\boldsymbol{\omega} & =\boldsymbol{\omega}(\boldsymbol{v}, \boldsymbol{E}, \boldsymbol{B}) \\
& =\boldsymbol{\Omega}_{1}(\boldsymbol{B}, v)+\boldsymbol{\Omega}_{2}(\boldsymbol{B}, \boldsymbol{v})+\boldsymbol{\Omega}_{3}(\boldsymbol{E}, \boldsymbol{v}), \\
\boldsymbol{\Omega}_{1} & =\frac{e}{m} \boldsymbol{B}\left(a+\gamma^{-1}\right)=-\omega_{L}(a y+1) \\
\boldsymbol{\Omega}_{2} & =-\frac{e}{m} \frac{(\boldsymbol{v} \boldsymbol{B})}{v^{2}} \boldsymbol{v}\left(1-y^{-1}\right) a \\
\boldsymbol{\Omega}_{3} & =-\frac{e}{m} \frac{\boldsymbol{v} \times \boldsymbol{E}}{c^{2}} \frac{a+y^{-1} g / 2}{1+y^{-1}},
\end{aligned}
$$

where $c$ is the velocity of light, $m$ is the rest mass of the electron, $e$ is the electron charge, $v=|\boldsymbol{v}|, y^{-1}$ $=\left(1-v^{2} / c^{2}\right)^{1 / 2}, \omega_{L}=-(e / m) y^{-1} B$ is the Larmor frequency, and $\omega_{L}=\omega_{L} \boldsymbol{B} / B, B=|\boldsymbol{B}|, g \approx 2.002$ is the $g$-factor of the free electron, $a=(g-2) / 2 \approx 10^{-3}$ is the $g$-factor anomaly.

According to (3) and (4a, b), $P$ is $P=$ const. for an ensemble of electrons with the same trajectory. For electrons with different trajectories, the vectors $\boldsymbol{P}_{\boldsymbol{i}}$ defined for partial ensembles $|i\rangle$ with the same trajectory will, in general, move differently. The average polarization has to be formed at each time $t$ according to (2). With (2) it follows that $P(t+\Delta t) \leqq P(t)$.

For $v \ll c$ (non-relativistic limit) $\boldsymbol{\Omega}_{2}$ and $\boldsymbol{\Omega}_{3}$ can be neglected relative to $\boldsymbol{\Omega}_{1}$. If, in addition, $\boldsymbol{B}$ is constant in time and space, the following solution of (3), $(4 a, b)$ is obtained

$$
\begin{aligned}
\boldsymbol{P}(t)= & \frac{\boldsymbol{P}_{0} \times \omega}{\omega} \sin \omega t+\boldsymbol{P}_{0} \cos \omega t \\
& +\frac{\omega\left(\omega \boldsymbol{P}_{0}\right)}{\omega^{2}}(1-\cos \omega t)
\end{aligned}
$$

where $P_{0}:=P(0)$ and $\omega=\omega_{L}(1+a) \approx \omega_{L}$.

For $\boldsymbol{P}_{0} \times \boldsymbol{\omega} \neq 0$ it is convenient to rewrite (5) by introducing the three orthogonal unit vectors

$$
\begin{aligned}
& \hat{\omega}:=\boldsymbol{\omega} / \omega=\boldsymbol{B} / B \\
& \hat{d}:=\hat{p}_{0} \times \hat{\omega} ; \quad \hat{p}_{0}=\boldsymbol{P}_{0} / P_{0} \\
& \hat{e}:=\hat{\omega} \times \hat{d}=\hat{p}_{0}-\hat{\omega}\left(\hat{\omega} \hat{p}_{0}\right) .
\end{aligned}
$$

This yields

$\boldsymbol{P}(t)=\left(\hat{e} \boldsymbol{P}_{0}\right)(\hat{d} \sin \omega t+\hat{e} \cos \omega t)+\hat{\omega}\left(\hat{\omega} \boldsymbol{P}_{0}\right)$,

This representation shows that $\boldsymbol{P}$ precesses about $\boldsymbol{\omega}$, i.e. about $\boldsymbol{B}$, with the frequency $\omega$. The component of $\boldsymbol{P}$ in the $\boldsymbol{\omega}$ direction is thereby constant. In the nonrelativistic limit a motion of $\boldsymbol{P}$ is therefore only caused by magnetic fields transverse to $\boldsymbol{P}$. The tilting angle

$\alpha:=\Varangle\left(\boldsymbol{P}(t), \boldsymbol{P}_{0}\right)$

thereby has the maximum value

$\alpha_{\max }=2 \cdot \Varangle(\boldsymbol{P}, \omega)=2 \arccos (\hat{\omega} \hat{p})$.

The following example illustrates the motion of $\boldsymbol{P}$ in a transverse magnetic field: for a homogeneous field with induction $B=0.1 \mathrm{mT}$ (order of magnitude of the earth's field) $\omega$ is $\omega=1.76 \times 10^{7} \mathrm{~s}^{-1}$. Let there be an ensemble of electrons moving in the field $\boldsymbol{B}$ with the velocity $v=0.1 c$ (energy $2.57 \mathrm{keV}$ ) and the initial polarization $P_{0}=1$ perpendicular to $\boldsymbol{B}$. The electrons transverse a distance of $1 \mathrm{~m}$ in $3.3 \times 10^{-8} \mathrm{~s}$; on this path $\boldsymbol{P}$ is tilted $0.58 \mathrm{rad}=33^{\circ}$. This motion is

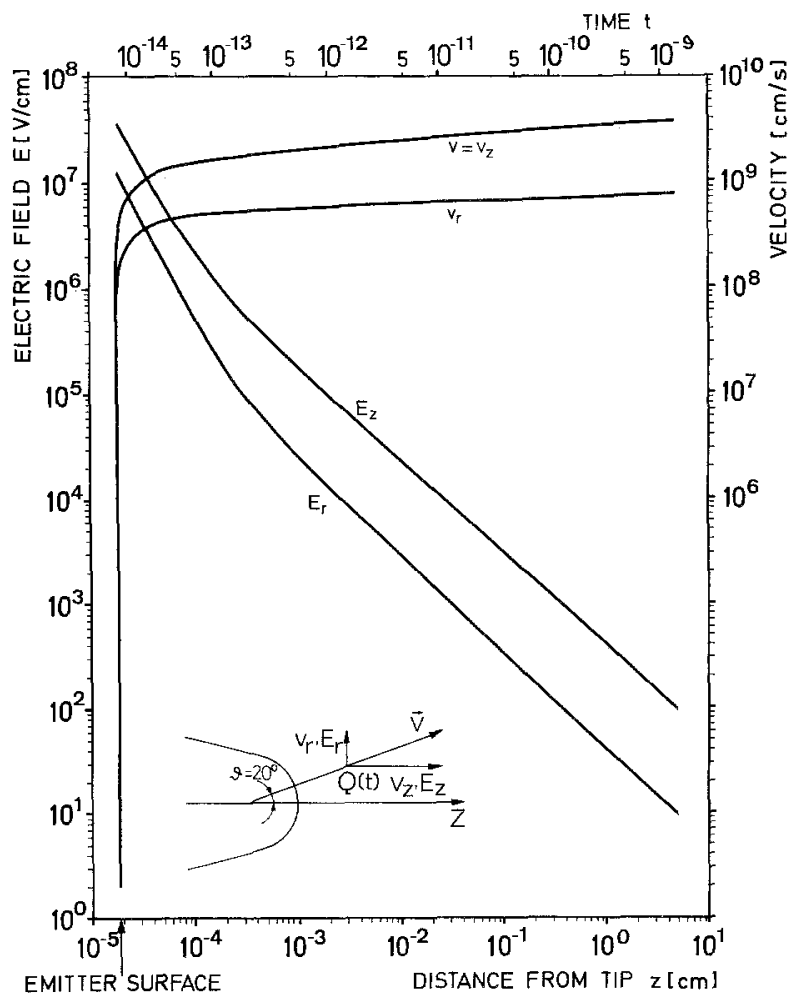

Fig. 1. Velocity and electric field strength along the trajectory of a field emitted electron 
independent of the direction of the velocity because $\boldsymbol{B}$ is homogeneous.

In field-emission experiments with polarized electrons the quantities governing $\omega$ in $(4 a, b)$ have the following limits: $B>10 \mathrm{mT}, v \leqq 0.15 \mathrm{c}, E<5 \times 10^{7} \mathrm{~V} / \mathrm{cm}$ (see Fig. 1). The terms $\boldsymbol{\Omega}_{2}, \boldsymbol{\Omega}_{3}$ in (3), $(4 \mathrm{a}, \mathrm{b})$ are therefore small relative to $\boldsymbol{\Omega}_{1}$. The foregoing remarks on the case of the non-relativistic limit thus also apply approximately to the motion of the polarization vector $\boldsymbol{P}$ in a FE experiment. Our calculations were made to determine the influence of $\boldsymbol{\Omega}_{2}, \boldsymbol{\Omega}_{3}$ and of a space dependence of $\boldsymbol{B}$. The interesting quantity in these calculation is the tilting angle because usually $\boldsymbol{B}_{0}$ and $\boldsymbol{P}_{0}$ are aligned in the symmetry axis of the emission arrangement. The precession about the symmetry axis is included in the calculations but is not shown in the results.

If $\boldsymbol{\Omega}_{2}$ and $\boldsymbol{\Omega}_{3}$ are not neglected and $\boldsymbol{B}$ is space dependent, $\boldsymbol{B}, \boldsymbol{E}$, and $\boldsymbol{v}$ have to be known for all points of individual electron trajectories in order to solve the equation of motion (3), $(4 a, b)$.

\section{Method of Calculation}

In the numerical treatment three types of fields rotational symmetric with respect to the $z$-axis are taken for $\boldsymbol{B}$. They are expressed by giving the axial component $\boldsymbol{B}_{z}(z)$ on the $z$-axis of a zylindrical coordinate system, $r, z$ :

Homogeneous field:

$B_{z}(z) \equiv B_{0}=$ const.

Bell-shaped field:

$B_{z}(z)=B_{0} /\left(1+z^{2} / D^{2}\right) ; D: B_{z}(D)=B_{0} / 2$.

Solenoid field:

$B_{z}(z)=B_{0}\left[1 / 4+R^{2} / L^{2}\right]^{1 / 2}$

$\left\{\frac{z+L / 2}{\left[(z+L / 2)^{2}+R^{2}\right]^{1 / 2}}-\frac{z-L / 2}{\left[(z-L / 2)^{2}+R^{2}\right]^{1 / 2}}\right\}$

with $R$ the radius and $L$ the length of the solenoid.

For points near the axis, this condition being well satisfied here, the radial component $B_{r}(r, z)$ is described by a series expansion according to [7]

$B_{r}(r, z)=-\sum_{\nu=1}^{\infty} \frac{(-1)^{v}}{v !(v-1) !}\left(\frac{r}{2}\right)^{2 v-1} B_{z}^{(z v-1)}(z)$.

In front of the tip $\boldsymbol{E}$ is essentially governed by the geometry of the emitter surface. For the tip shape we

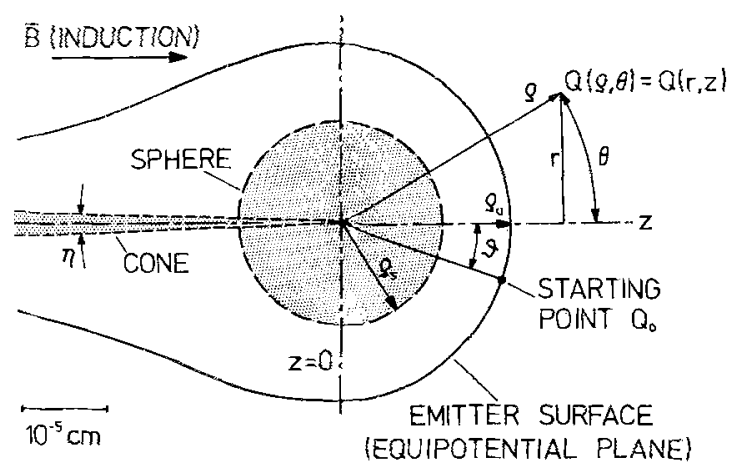

Fig. 2. Tip-model according to Dyke et al. [8]

use the model of Dyke et al. [8]: The emission surface is an equipotential surface which is formed in front of a cone with an apex angle $\eta$ whose point is the centre of a sphere of radius $\varrho_{s}$ (see Fig. 2). The electric potential of this sphere-on-orthogonal-cone is zero. $V_{a}$ is the electric potential of an equipotential surface serving as anode at a pole distance of $\varrho_{a} \gg \varrho_{s}$ from the centre of the sphere. The potential $V$ of $E$ is then given in plane polar coordinates $\varrho, \Theta$ with the centre of the sphere as origin and the cone direction as zero direction (see Fig. 2) by

$V(\varrho, \Theta)=\left(V_{a} / \varrho_{a}\right)\left(\varrho^{n}+\varrho_{s}^{2 n+1} \varrho^{-(n+1)}\right) P_{n}(\cos \Theta)$

where $P_{n}(\cos \Theta)$ is the Legendre function with $n$ chosen such that the function vanishes for $\Theta=\pi-\eta / 2$ (generally $n$ non-integral).

To get the surface

$V(\varrho(\Theta), \Theta)=V\left(\varrho_{0}, 0\right)=$ const.

of a typical FE tip, according to Vernickel and Welter [9] the following parameters are used

$\varrho_{0}=2 \times 10^{-5} \mathrm{~cm}$

$\varrho_{s}=1.2 \times 10^{-5} \mathrm{~cm}$

$n=0.11$

$\varrho_{a}=5 \mathrm{~cm}$

$V_{a}=V\left(\varrho_{a}, 0\right)=4.31 \times 10^{3} \mathrm{~V}$.

Thus $V(\varrho, \Theta)$, and hence $E(\varrho, \Theta)=-\operatorname{grad} V$ [and the shape $V(\varrho(\Theta), \Theta)=V_{a}$ of the anode], are determined. The emitter potential is $V\left(\varrho_{0}, 0\right)$.

We obtain $v(z)$ and the electron trajectories $r(z)$ $=(x(z), y(z))$ by integrating the equation of motion

$\frac{d \boldsymbol{p}}{d t}=\frac{d}{d t}(m \gamma \boldsymbol{v})=e(\boldsymbol{E}+\boldsymbol{v} \times \boldsymbol{B})$. 
As $V_{0}<5 \mathrm{kV}$, we get $v / c<0.15$ and $\gamma-1<0.012$. Equation (15) can therefore be solved in the nonrelativistic approximation $\gamma=1$.

Numerical integration of (15) and (3), $(4 a, b)$ was performed by a predictor-corrector method. The initial conditions were as follows:

1) The electrons start with velocity $v_{0}=0$ on the equipotential surface (14a) given above.

Except for a rotation about the symmetry axis of the field ( $z$-axis), the starting points $Q$ on (14a) are given by the starting angle $\delta . Q$ and the $z$-axis define the starting plane. They are always located in the $x-z$ plane of a Cartesian coordinate system whose origin is in the centre of the emitter sphere (see Fig. 2).

2) The centre of symmetry of the magnetic field is usually located in the origin of the coordinate system, the axis of symmetry in the $z$-axis; to investigate the influence of misalignment, the position of the magnetic field can be shifted relative to the tip.

3) At the starting point $Q \boldsymbol{P}_{0}$ is in the $z$-direction. The tilting of $P(t)$ relative to $\boldsymbol{P}_{0}$ is given by $\propto$ defined in (8) or by $\operatorname{tg} \alpha$.

The magnetic induction $B_{0}$ in the centre and the starting angle $\vartheta$ are varied: $B_{0}$ from 0 to $5 \mathrm{~T}$ and $\vartheta$ from $0^{\circ}$ to $45^{\circ}$. The selected interval for $\vartheta$ is typical of the emission cone of a FE tip [10].

\section{Results and Discussion}

a) Ideal Adjustment of the Field Axes, $\boldsymbol{P}_{0}$ Aligned with $\boldsymbol{B}_{0}$

Results of the calculations are shown in Fig. 3-6: In the non-relativistic case, neglecting $\boldsymbol{\Omega}_{2}, \boldsymbol{\Omega}_{3}$ in (4a), there ought not to be any tilting of $\boldsymbol{P}$ in the homogeneous magnetic field under the given starting conditions $\left(\boldsymbol{P}_{0} \| \boldsymbol{B}_{0}\right)$. Figure 3 shows the rotation calculated with allowance for $\boldsymbol{\Omega}_{2}, \boldsymbol{\Omega}_{3}$ for various types of magnetic field and the starting angle $\vartheta=20^{\circ}$ (a mean starting angle). The tilting at $B(z) \equiv 0$ is ascribed to the influence of the electric field term $\boldsymbol{\Omega}_{3}$, the tilting at the homogeneous field $B(z)=B_{0}$ to the influence of $\boldsymbol{\Omega}_{2}$ and $\boldsymbol{\Omega}_{3}$. The tilting in both cases is less than $0.1^{\circ}$ and therefore negligible. The deviations from the homogeneous field case occur with the other types of fields, the bell-shaped and solenoid field, when transverse field components $B_{r}$ become effective. This behaviour is seen in Fig. $4: B_{r}(z)$ has maximum values at the maxima of the distance $r(z)$ of the electron trajectories from the $z$-axis. At these points $\alpha$ increases stepwise.

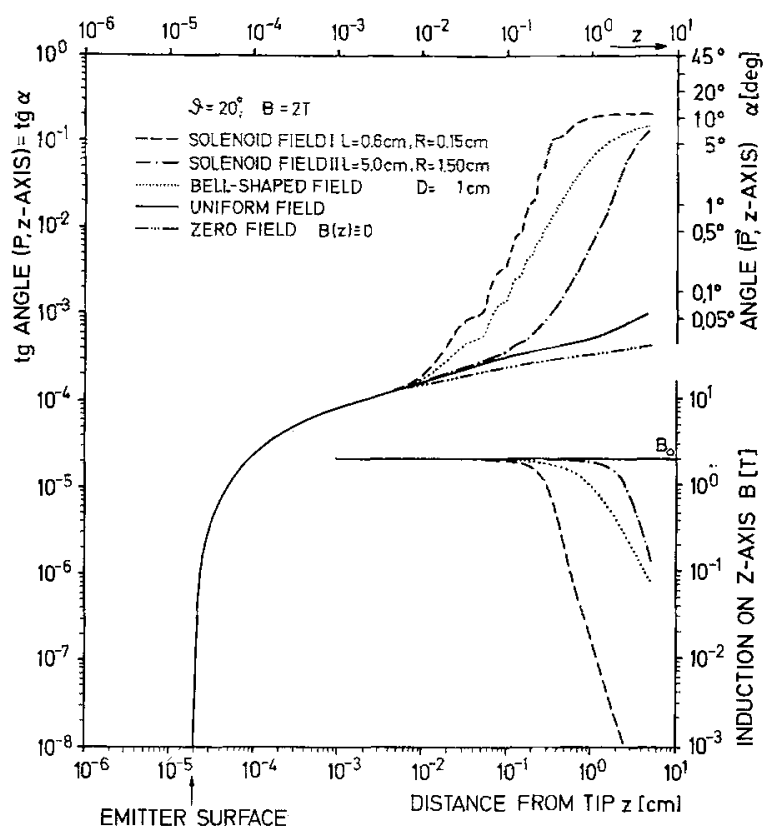

Fig. 3. The tilting angle $\alpha$ of the polarization vector $\boldsymbol{P}$ against the initial direction ( $z$-axis) for different magnetic field configurations as a function of the distance $z$ from the tip. The solenoid field $I$ is similar to the configuration used in the experiment of $[1,2,4]$, the Solenoid Field II corresponds to the arrangement used in [3] neglecting magnetic shielding. The bell-shaped field can be regarded as a field of a solenoid with special shielding. The influence of the electric field on $\alpha$ is shown in the curve with $B=0$

Figure 5 shows a surprising result: $\alpha$ only depends slightly on the magnetic induction $B_{0}$ in the centre, if one neglects the scattering of $\alpha$ at low values of $B_{0}$ (the reason for this scattering, especially at Solenoid I, is that the periodicity in $r(z)$ is of the order of the solenoid length). On the other hand, the dependence on the starting angle $\vartheta$, shown in Fig. 6, is strong. This behaviour is due to the fact that the maxima of $r(z)$, and hence the maxima in $B_{r}(z)$, become larger with decreasing $B_{0}$ and increasing $\vartheta$ (see Fig. 7). The cited results are performed for a tip with constant radius $\varrho_{0}=2 \times 10^{-5} \mathrm{~cm}$. If $\varrho_{0}$ decreases (increases) $E(\varrho, \Theta)$ is changed, and if $B_{0}$ is constant the electron trajectories are shifted to smaller (larger) $r(z)$. Correspondingly the tilting of $\boldsymbol{P}$ decreases (increases). For comparison, in Fig. 7 the maxima of $r(z)$ and of $B_{r}(z)$ and $\alpha(z)$ are also given for a smaller tip and the same electric field strength at the tip $\varrho_{0}=3.7$ $\times 10^{-6} \mathrm{~cm}, \varrho_{s}=2.0 \times 10^{-6} \mathrm{~cm}, n=0.21, V_{a}=3.86$ $\times 10^{3} \mathrm{~V}$ ), a tip typical of field ion microscopy. A decrease (increase) of the work function should have a similar effect as the decrease (increase) of $\varrho_{0}$ mentioned above, because for a constant emitter current a work function change can be simulated by a change of $\varrho_{0}$ 

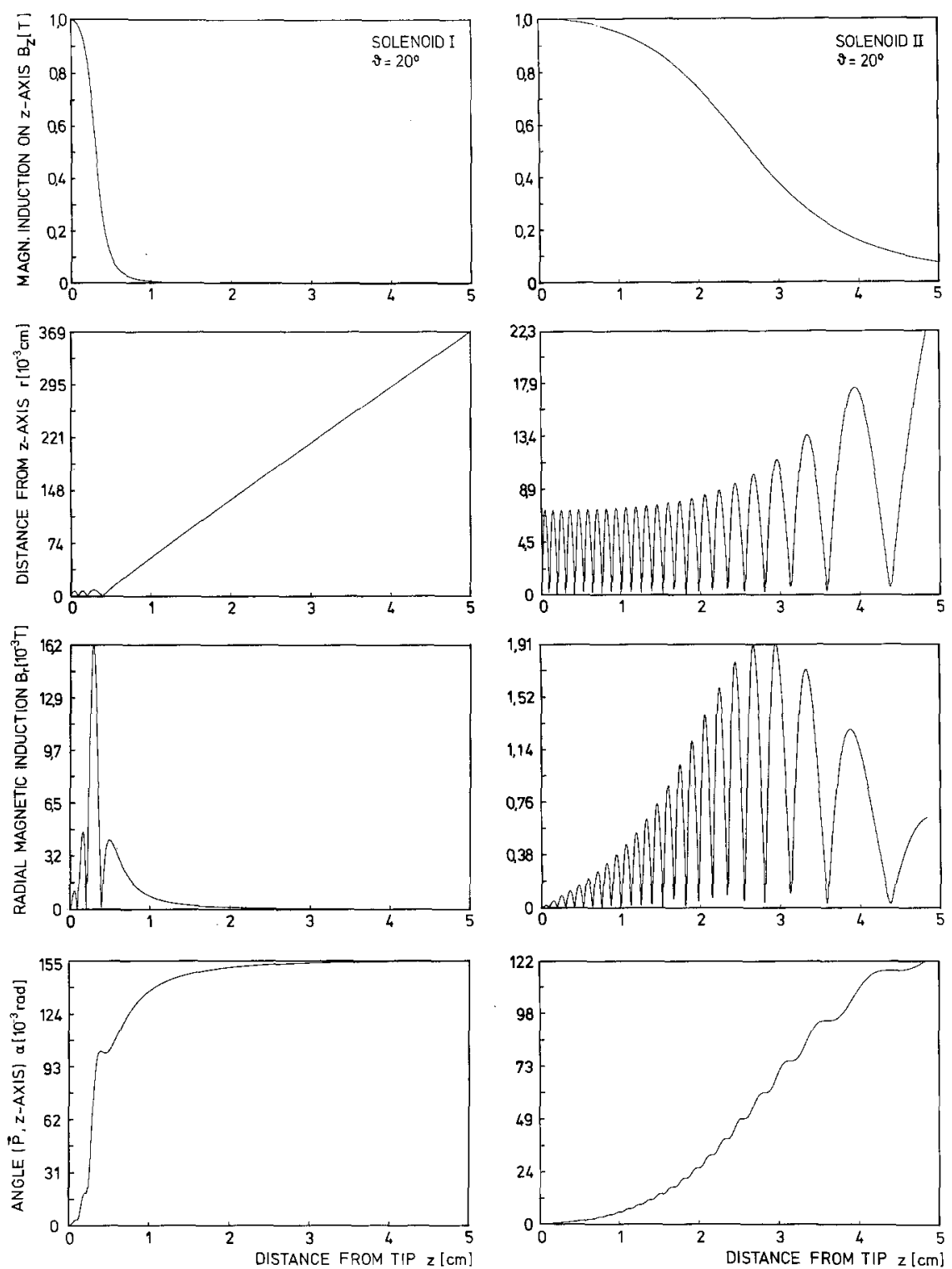

Fig. 4. Magnetic induction $B_{z}(z)$ on the $z$-axis, distance $r(z)$ of the electron trajectory from the $z$-axis, radial component $B_{r}(z)$ of the magnetic induction on the electron trajectory, tilting $\alpha(z)$ of the polarization vector $\boldsymbol{P}$ against his initial direction $(z$-axis)

with the other constants fixed and because an initial velocity $v_{0}$ of the order of $10^{8} \mathrm{~cm} \mathrm{~s}^{-1}$ is not important. Calculations with $v_{0}=v_{F}$ (the Fermi velocity) have shown that initial velocities of this order can be neglected.

b) Misalignment of the Field Axes, Magnetization Errors

First it is postulated that $\boldsymbol{P}_{0}$ has no component perpendicular to $\boldsymbol{B}_{0}$ (no magnetization error). Misalignment of the axes of the electric and magnetic field relative to one another will have an effect when the electron trajectories are thereby shifted to regions with larger $B_{r}$. Correspondingly, there are no noticeable effects in the motion of $\boldsymbol{P}(t)$ when the tip axis (the electric field axis) is tilted towards the magnetic field axis and the centre of the tip comes to rest on the magnetic field axis. The position of the starting points and the electric field relative to the magnetic field thereby does not appreciably change, i.e. the trajectories are only slightly shifted compared with the case of ideal adjustment. 


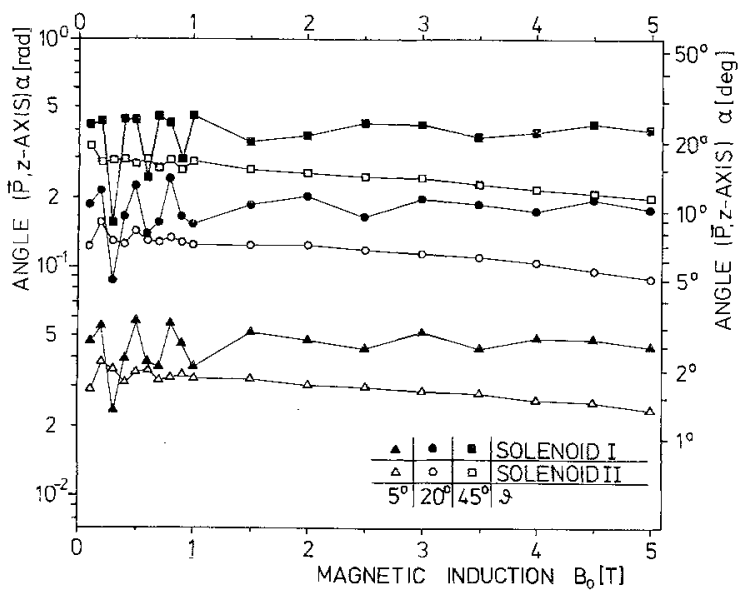

Fig. 5. Tilting angle $\alpha$ of the polarization vector $P$ at a distance $z=5 \mathrm{~cm}$ from the tip against his initial direction (z-axis) as a function of the magnetic induction $B_{0}$ in the field center for starting angles $9=5^{\circ}, 20^{\circ}, 45^{\circ}$ and for Solenoids I and II

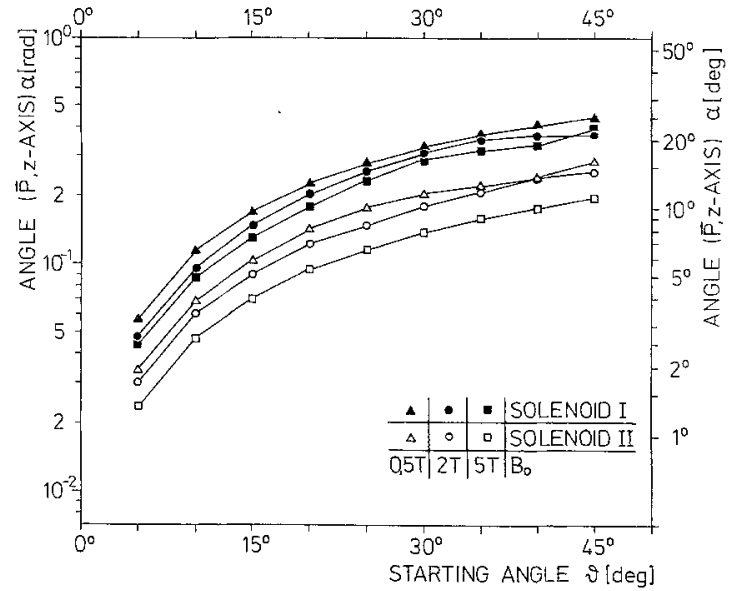

Fig. 6. Tilting angle $\alpha$ of the polarization vector $P$ at a distance of $z=5 \mathrm{~cm}$ from the tip against his intial direction (z-axis) as a function of the starting angle $\vartheta$ for the magnetic inductions $B_{0}=0.5,2,5 \mathrm{~T}$ in the center of the Solenoids I and II
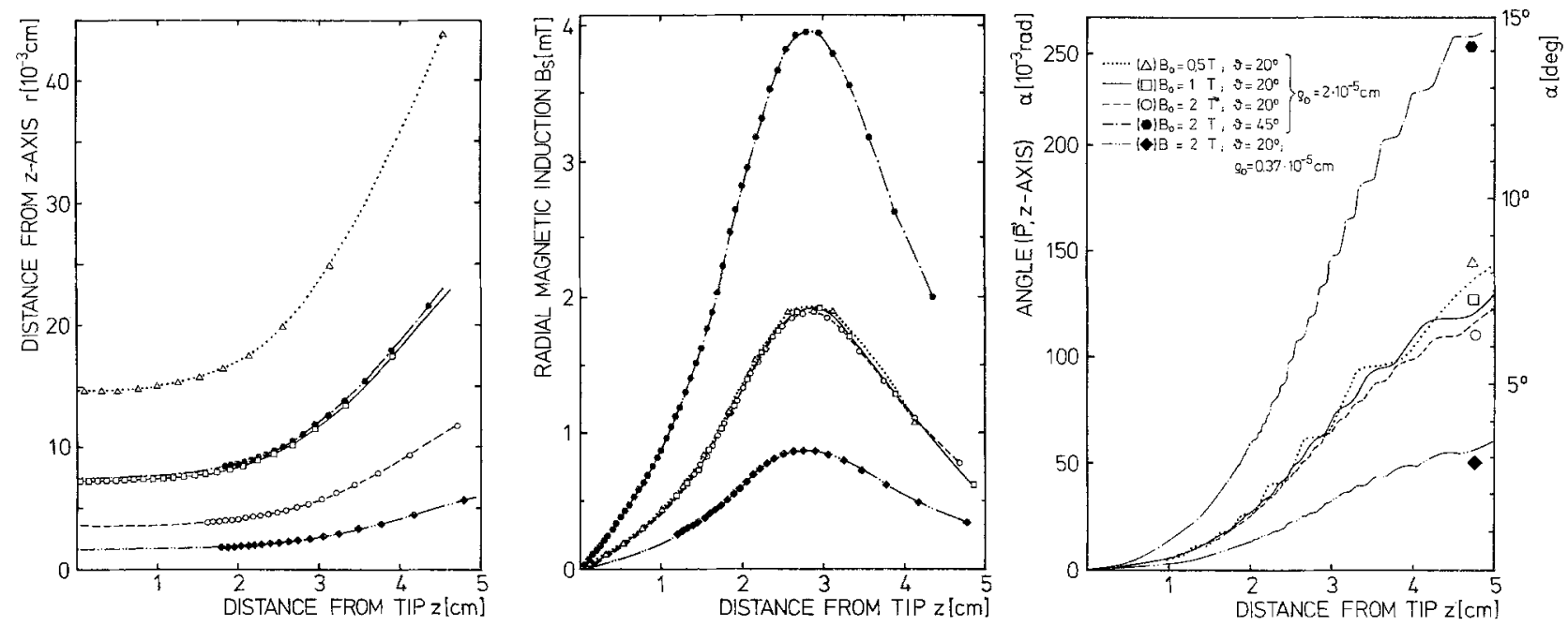

Fig. 7. Comparison of distances $r(z)$ of the electron trajectories from the $z$-axis, of radial magnetic inductions $B_{r}(z)$ along the trajectories, and of the resulting tilting angle $\alpha(z)$ for the Solenoid II with variations of the starting angle $\vartheta$, of the magnetic induction $B_{0}$, and of the tip radius: For $r(z)$ and for $B_{r}(z)$ the enveloppes of the curves in Fig. 4 are given. The points indicate the maxima in the curves of Fig. 4 (as far as these points are not to near together)

Critical, on the other hand, is lateral misalignment, i.e. lateral shifting of the tip axis towards the magnetic field axis. The electron trajectories are shifted to regions with larger $B_{r}$. Figure 8 shows the influence of a shift of the tip in the symmetry plane of two solenoid fields. Particularly in the case of the small coil (Solenoid I) lateral misalignment of a quantity that is realistic for experiments leads to appreciable tilting of $\boldsymbol{P}$. This effect will be enlarged at higher magnetic inductions.
If $\boldsymbol{P}_{0}$ has a component $\boldsymbol{P}_{\boldsymbol{e}}$ perpendicular to $\boldsymbol{B}_{0}$ (magnetization error), $\boldsymbol{P}$ precesses about $\boldsymbol{B}_{0}$ according to (7). The influence of $\boldsymbol{\Omega}_{2}, \boldsymbol{\Omega}_{3}$ is negligible for a not too small transverse component $\boldsymbol{P}_{e}$.

In the calculations it is always the motion of the polarization vector $\boldsymbol{P}$ for rays emanating from individual points of the emitter that is determined. If the whole emission cone of a FE tip is considered in an experiment, as mentioned above, it is necessary to average over the emission characteristics of the 


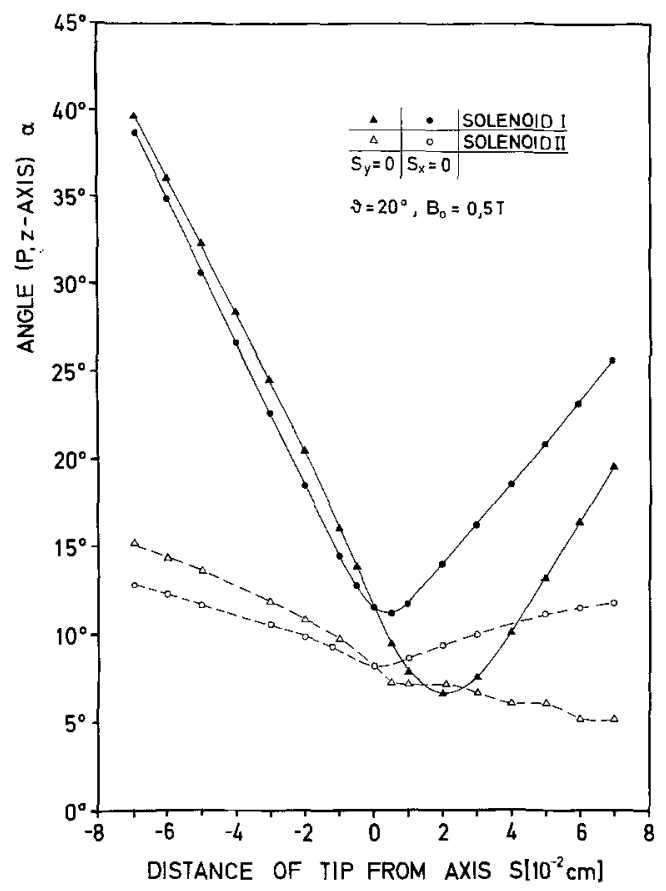

Fig. 8. Influence of a radial misalignment $S=\left(S_{x}, S_{y}\right)$ of the tip on the tilting angle $\alpha$ at a tip distance $z=5 \mathrm{~cm}$ for a magnetic induction $B_{0}=0.5 \mathrm{~T}$ in the center of the Solenoids I and II

emitter according to (2) in order to estimate the depolarization $\left(1-\langle P(t)\rangle / P_{0}\right)$ and the mean tilting angle $\langle\alpha\rangle$ of the average polarization vector $\langle\boldsymbol{P}(t)\rangle$ relative to $\boldsymbol{P}_{0}$.

\section{Conclusions}

To summarize, it can be stated that the ESP in FE experiments is not appreciably influenced by the electric and magnetic fields in front of the FE tip when there is magnetic saturation of the emitter $\left(\boldsymbol{P}_{0}\right.$ and $\boldsymbol{B}_{0}$ aligned) and sufficiently good centring of the FE tip in the magnetic field. This agrees with the results of the experiments by Müller et al. [3].

The authors wish to thank V. Welge for her work with the program, C. Fritsch, M. Krebs, W. Ottenberger for preparing the drawings, and A. McAllan Nicol for the English translation. We are grateful. to $\mathrm{H}$. J. Meister and $\mathrm{H}$. Vernickel for useful discussions and critical reading of the manuscript.

\section{References}

1. M.Hofmann, G.Regenfus, O.Schärpf, P.J.Kennedy: Phys. Letters 25A, 270(1967). G. Chrobok, M. Hofmann, G. Regenfus: Phys. Letters 26A, 551 (1968)

2. W. Gleich, G. Regenfus, R. Sizmann: Phys. Rev. Letters 27, 1066 (1971)

3. N.Müller, W.Eckstein, W.Heiland, W.Zinn: Phys. Rev. Letters 29, 1651 (1972)

4. G. Regenfus, P.Sütsch: Z. Physik 266, 319 (1974)

5. M.Kolsrud: Nuovo Cimento 39, 504 (1965)

6. Equation $(4 a, b)$ is given in the notation used in the review article by P. S. Farago (Advan. Electron. Electron Phys. 21, 1 (1966), chap. III). The printing errors in Faragos eq. III, (25) are corrected here. Printing errors also occur in the corresponding equation $(3,5)$ of the review article by A. Rich and J. C. Wesley (Rev. Mod. Phys. 44, 250 (1972). - (4a, b) of this work is identical with eq. (17.18) in the paper of D. M. Fradkin and R. H. Good (Rev. Mod. Phys. 33, 343 (1961)) and with eq. (17) in the paper of H. J. Meister (Z. Physik 166, 468 (1962)).

7. W. Glaser: Grundlagen der Elektronenoptik (Springer-Verlag, Wien 1952) p. 144

8. W.P.Dyke, J.K.Trolan, W.W. Dolan, G.Barnes: J. Appl. Phys. 24, 570 (1953)

9. H.Vernickel, H.Welter; Internal Report IPP $7 / 2(6 / 88)$, Institut für Plasmaphysik, Garching (1970)

10. M.Drechsler, E. Henkel: Z. Angew. Physik 6, 22 (1954) 